\title{
Modeling Influencing Factors for Passenger Flow Growth of Modern Trams Using System Dynamics Method
}

\author{
Mao Ye (D), ${ }^{1}$ Ninghui Yang, ${ }^{2}$ Zhibin Li $\mathbb{D}^{,},{ }^{3}$ Lingling Ma, ${ }^{1}$ and Yajing Chen ${ }^{1}$ \\ ${ }^{1}$ Department of Traffic and Transportation Engineering, School of Automation, Nanjing University of Science and Technology, \\ 200 Xiao Ling Wei, Nanjing, Jiangsu 210094, China \\ ${ }^{2}$ School of Materials and Engineering, Yancheng Institute of Technology, Yancheng, Jiangsu 224051, China \\ ${ }^{3}$ School of Transportation, Southeast University, 2 Si Pai Lou, Nanjing, Jiangsu 210096, China \\ Correspondence should be addressed to Mao Ye; yemao0924@163.com
}

Received 30 December 2018; Accepted 1 August 2019; Published 25 November 2019

Academic Editor: Guohui Zhang

Copyright (C) 2019 Mao Ye et al. This is an open access article distributed under the Creative Commons Attribution License, which permits unrestricted use, distribution, and reproduction in any medium, provided the original work is properly cited.

\begin{abstract}
Modern trams have been widely used around the world, especially in China. This paper explores the main influencing factors of modern trams' passenger flow at the early operational stage. The system dynamics model is adopted for dealing with the problem on hand. Tram Line 1 in Huai'an, Jiangsu Province, China is selected as the case study. Data are collected using the RP and SP survey. The sensitivity test and extreme condition test are performed. The simulation results demonstrate that four variables (i.e., land development intensity, fares, service level, and transfer efficiency) significantly affect passenger flow. Land development intensity is the most significant factor, and the effect of service level on passenger flow is higher than that of the fares. The departure interval of 10 minutes is the maximum psychological limit that passengers can bear, and $2 \mathrm{RMB}$ is a reasonable price. Such conclusions can provide guidance for the planning and design of modern trams and address the problem of shortage of passengers at an early stage.
\end{abstract}

\section{Introduction}

The modern tram is a low-floor electric vehicle guided by electric traction wheel rails. It runs on a dedicated track and is a medium and low-volume rail transit system with levelcrossings and priority signals [1]. As a low-cost, green, convenient, and reliable rail transit, trams have been widely constructed in many countries around the world [2-4]. Throughout the development of trams, since the construction of the first tram in Germany in the 1880s, trams have experienced three stages: rapid development stage, gradual decline stage, and then rapid rise stage [4]. With the advancement in technology, the old-style trams with mixed road rights, low speed, noise, poor comfort, and low flexibility have gradually developed into modern trams with special road rights, fast speed, good comfort, and flexible grouping.

At present, the modern tram system has become an important part of the urban passenger transportation system in many countries. According to incomplete statistics, more than 400 lines have been constructed in the world, and the total mileage is more than 4,000 kilometers. In addition, the total number of trams is more than 6,000 , and the daily passenger volume is about 30 billion. Modern trams have become the backbone of public transport in many underdeveloped areas, new regions, or outer space in large cities, and small and medium-sized cities, especially in China [5]. Statistics showed that, as of the end of 2017, nearly 100 cities (including Beijing, Shanghai, Nanjing, and Huaian) in China have been planning a total of more than $10,378 \mathrm{~km}$ of tram lines. The mileage under construction is up to $585.56 \mathrm{~km}$, and the operating mileage that has been opened up is about $232.7 \mathrm{~km}$ [6].

The core objective of the modern tram system is to provide residents with high-quality public transport services, and therefore, scientific planning of modern tram lines and the development of a reasonable operational organization are essential. A key factor that needs to be taken into account in the planning, construction, and operation of trams is the passenger flow [7]. For the construction cost or operational efficiency of the tram, the passenger flow is inseparable $[8,9]$. The construction of tram network in many abroad cities has almost been completed, and the operating organization is relatively stable. However, many cities in China are in the rapid 
construction period of the tram network. Such cities have only started construction and operation in recent years. Currently, there are only 1-2 lines in some cities, which are basically in the single-line operational stage.

The passenger volume forecasting model is often used to estimate and analyze the passenger flow of trams. Since the passenger flow volume is affected by various factors, precise forecasting results are always difficult to obtain. According to existing studies [9-16], the passenger flow of trams can be affected by land development along the line, line size, transfer efficiency, service level, and ticket prices. The passenger volume of trams is not high since the modern tram has just come into service in China. For example, the daily average passenger flow (DAPF) in Nanjing Hexi New City Line is 2,900 passengers/day; the DAPF in Nanjing Qilin Line 6900 passengers/ day; the DAPF in Guangzhou Haizhu Line and Suzhou Hightech Zone Line 1 is 6,700 passengers/day and 7,500 passengers/ day, respectively. Low passenger flow has become an important constraint to the development of trams. The main reasons are summarized as follows: (1) Currently, for most cities in China, trams are in the single-line operational stage, which has not yet been networked and has limited service area; (2) Trams have not been put into operation for a long time, which is still in the initial operational stage; (3) Due to the impact of land use, line connection, transfer facilities, and service levels along the line, passenger flow is difficult to meet expectations. As a result, it is essential to explore and analyze the influencing factors which affect the growth of trams passenger flow.

There are two core objectives in this paper: (1) systematically analyzing the typical influencing factors and causality of modern tram passenger flow; (2) adopting the system dynamics model to study the impact of factors on passenger flow. In addition, a sensitivity test is also performed. The simulation results can provide a basis for passenger flow improvement. Research results can provide a reference for modern tram planning and passenger flow forecasting, and provide guidance for solving low passenger flow in the early operational stage of modern trams.

This paper is organized as follows. Section two presents the literature review. Section three describes the formulation of the system dynamics model. Section four performs the data description and analysis. Section five presents the simulation results. The final section includes the conclusion and discussion of future work.

\section{Literature Review}

Promising achievement on trams has been obtained. The existing research efforts mainly focus on the utility of trams, impact on land use, and residents' travel, traffic operation, and traffic signal priority design.

$\mathrm{Zi}$ et al. [17] proposed that modern trams were suitable for the following three types of regions in China: main public transport system in new town and satellite city in the metropolis, peripheral areas where are not covered by rail transit services, and backbone public transportation system in the medium and small city. Termida et al. [18] selected a new tram line in Stockholm as the case study. The authors conducted a four-wave survey to collect personal travel and psychological attribute data. They studied the dynamic response of residents' travel behaviors and patterns by using the mixed logit model and panel data. By analyzing the travel mode selection and comparing models and inertia effects, González et al. [19] proposed that the asymmetric effect between private cars and public transportation would increase after the construction of trams. Gadziński and Radzimski [20] collected data on the operation of the first tram line in Poland from 2010 to 2013. The spatial econometric model and weighted regression model were used to analyze how the route affected residents' travel behavior, housing choice, satisfaction, and housing prices.

In the area of analysis of passenger flow and influencing factors of urban public transportation, Chatterjee and $\mathrm{Ma}$ [21] proposed that the response of individual varies when introducing a new public transport service. The results of this study demonstrated that travelers' perception and use of new bus services varied over time. The authors used the duration model to explore the influencing factors and provide support for improving passenger flow forecasting and operational services. Though exploring the influencing factors of passenger flow at the subway station, Pan et al. [9] found that passenger flow was related to the employment density around the station and the commuting distance of residents. Passenger flow had a positive correlation with the same factors, such as the start time of the first train and whether a station is a transfer station. The passenger flow was always large at the stations where the business was well developed. Walton and Sunseri [22] analyzed the sample data of drivers and walkers arriving at the public transportation facilities and found that parking and transfer were affected by ten major factors. Among these factors, the convenience of parking lot affected the parking and transfer demand, and thus affecting the passenger flow. González et al. [14] studied the factors that could be used to explain the distorted perception of the tram's travel time. The results indicated that the convenience of the tram, the frequency of service, and waiting time affected the choice of trams. Increasing the convenience and the frequency of departures and reducing the waiting time could greatly attract passengers. Paulley et al. [23] studied factors affecting the use of public transport which mainly focus on the fare, quality of service, household income, and car ownership. Huang et al. [24] developed a distance-based nonlinear bus fare structure. Based on the game between the transport authority and operating company and the passengers, the optimal fare function and service frequency were determined, and an optimization model was formulated.

System dynamics is a discipline that studies information feedback systems, which analyzes and processes complex system problems by combining qualitative and quantitative methods. Since the 1990s, this method has been widely used in transportation, logistics and supply chain management, urban development and ecosystems, and operations research fields. Shepherd [25] systematically explained the application of system dynamics in transportation systems and summarized and proposed the key issues of the application of this method in the transportation field. Besiou et al. [26] used the system dynamics method to establish a causal relationship diagram 
of the whole process chain including materials acquisition, transfer, transportation, use, and demand changes to reveal the dynamic relationship of emergency materials allocation. Liu et al. [27] adopted system dynamics to establish a framework for congestion charging, providing decision makers with an evaluation platform to understand regional congestion charging policies and other short-term and longterm system behaviors caused by congestion mitigation policies. Yang et al. [28] developed a system dynamics model for managing urban traffic land to provide guidance for countermeasure development. Li et al. [29] employed the system dynamics method to study the main factors of the speed of emergency materials transportation from the perspective of government emergency management.

\section{Methodology}

3.1. Modeling Framework. The modern tram transportation system is a feedback system involving multi-variable, highorder, multiloop, nonlinearity, and strong nonlinearity in the fields of society, economy, and nature. The state variable (i.e., passenger flow) is affected by certain factors which change over time. Changes in these factors will likely result in changes in state variables. Due to the complexity of the system, some parameters are difficult to quantify.

The essence of the analysis of the influence factors of passenger flow is to predict the passenger flow of modern tram, which provides the basis for line orientation, site setting, capacity allocation, and departure plan. The commonly used methods for analyzing the influence factors of passenger flow include, but are not limited to, linear regression model [30] et al. Due to the numerous influencing factors as well as the complicated relationship between them, accurate prediction and analysis results are always difficult to achieve. Thus, more important is the trend analysis. Compared to traditional qualitative and quantitative analysis methods, the system dynamics method qualitatively analyzes the relationship between the influencing factors and the passenger flow and also quantifies these relationships by constructing dynamic equations. The Vensim is used to achieve dynamic simulation relationship. Therefore this method is used in this paper.

The following processes are included when using system dynamics to analyze the impact factors on trams' passenger flow: collecting influencing factors (survey), constructing system causality loop diagram and flowchart (feedback system analysis), system modeling, and simulation and sensitivity analysis. The modeling flow chart is shown in Figure 1.

3.2. Analysis of System Causality. Most of the objects studied by the system dynamics are high-order systems formed by the interaction of multiple factors. The essence is to decompose complex high-order systems into several low-order subsystems and to reverse the evolution mechanism of higherorder systems by studying the functions of internal subsystems and the connections between subsystems. The order is the number of state variables in the system. The first-order feedback system is the most basic system of system dynamics model. S-type growth system belongs to the first-order system with multiple feedback, including a positive feedback loop and a negative feedback loop. S-type growth is a typical system behavior, including both the exponential and asymptotic growth processes.

Feedback loop is a closed loop consisting of a series of causal and interaction chains, which can be divided into positive feedback and negative feedback. The characteristics of positive feedback loop are as follows: it can produce the strengthening process of its own motion, in which the consequences caused by movement or action will be reciprocated and the original trend will be strengthened; The characteristic of negative feedback loop is that it can automatically seek a given goal, and will respond continuously when it fails to reach (or does not approach) the goal. As shown in Figure 2, the curve of passenger flow growth and change is quoted from reference [31-33], describing the characteristics of passenger flow changing with time. The vertical coordinate represents the passenger flow Volume, the arrow to the right of abscissa represents the time $t$, and the arrow to the left represents the passenger flow change rate TC.Rate, which can be expressed as:

$$
\frac{d(\text { Volume })}{d t}=\text { TC.Rate } * \text { Volume } *\left(1-\frac{\text { Volume }}{\text { V.max }}\right) .
$$

In formula (1), V.max represents the maximum capacity of the system, that is, the stable value.

In the early stage of modern tram operation, Volume is small, 1 - Volume/V.max is close to 1, and its development is in an unconditional restrictive state. TC.Rate keeps increasing, forming an exponential growth process of positive feedback until it reaches a certain value, that is, inflection point. When the modern tram develops to a certain scale, it turns into negative feedback area. The increase of passenger flow is restricted by social, economic, population, traffic environment and the conditions of tram itself. Volume continues to grow. However, TC.Rate is decreasing until it tends to 0. Accordingly, Volume asymptotically reaches the stable value V.max of the system, and the development of passenger flow reaches a stable equilibrium state. The whole development presents an S-shaped growth curve. S-type growth belongs to the first-order system with multi-feedback, which includes a positive feedback loop and a negative feedback loop. The necessary condition for S-type growth characteristics is that the dominant feedback loop in the system changing, and the exponential growth process is dominated by the positive feedback loop, while the asymptotic growth is dominated by the negative feedback loop.

The modern tram transportation system is a complex high-order system, but in the specific research, it is usually possible to decompose high-order problems of multiple factors into several first-order system problems. The growth of things will go through three stages of occurrence, development, and maturity. The development speed and performance characteristics of each stage are different. The change in the occurrence phase is slow; the change in the development phase is accelerated; the change in the mature phase tends to be flat, and the curve obtained according to this law is the growth curve. The growth process of urban rail transit passenger flow follows the growth law and is generally on the rise. 


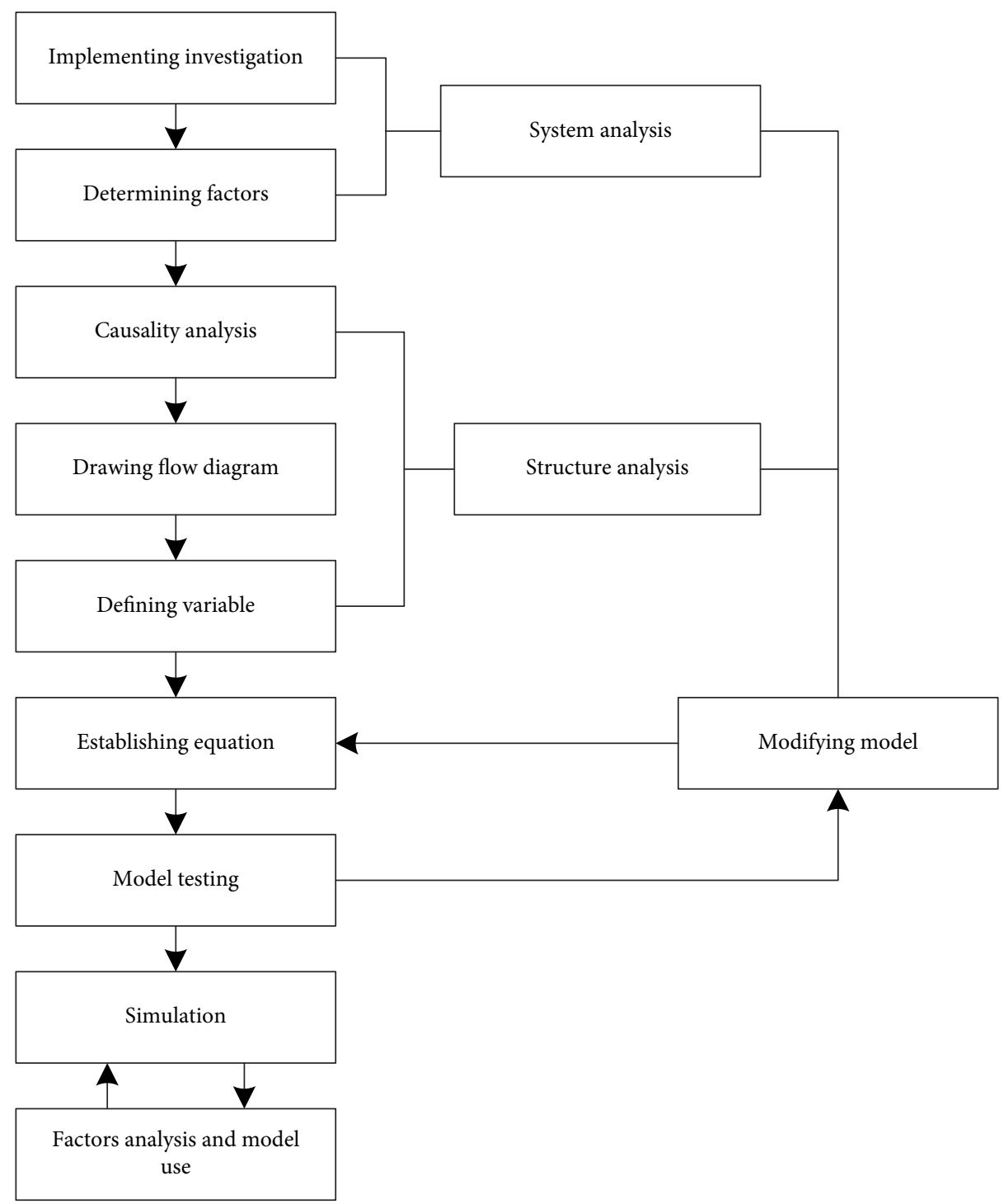

FIGURE 1: Modeling framework of system dynamics.



FIGURE 2: Curve chart of passenger flow growth and change of modern tram. 
According to the research results in [34-36], different growth characteristics are presented in different operational stages, which can be roughly divided into three stages: The initial operation is the stage of passenger flow cultivation, which mainly refers to the time of the first 3 years after the opening of urban rail transit. At this time, the construction of urban rail transit has just started, and there are few operating lines. It needs to experience a process of residents' cognition, adaptation, and familiarization, and finally form a reasonable passenger flow attraction area. At this stage, the passenger flow grows slowly. The second stage is the growth period, usually around 10 years. With the gradual maturity of urban rail transit construction and operation, the increase of residents' income level, urban population growth, road traffic congestion, and changes in residents' travel habits, passenger flow development during this period is in an unconditional highspeed growth stage. The third stage is the stable period. Network construction enters the network stage, which is restricted by the social, economic, population, and transportation environment, as well as the constraints of the rail transit itself. The index growth has turned into gradual growth. With the increase in the total utilization rate, the passenger flow gradually approaches the maximum capacity, and the rate of change of passenger traffic gradually becomes zero and enters a stable state.

It can be seen that the changes in passenger flow of modern trams are the result of positive feedback and negative feedback at different development stages. In the initial operational stage, the main role is to meet demand and relieve the passenger pressure. When trams develop to a certain scale (negative feedback plays a leading role), the passengers' perceived utility will affect the choice of travel mode. The increase in passenger flow will result in the reduction of service level, which will lead to a decrease in passenger growth rate, transformed into asymptotic growth. The current operation is still short, which is in the initial stage of exponential growth, but does not rule out the rapid growth of special time periods.

According to the research results in the literature [35], the passengers of modern tram mainly consist of three parts: growth passenger flow, induced passenger flow, and transferred passenger flow. The growth passenger flow is the passenger flow that needs to be satisfied in the initial stage of the construction of trams, and its size is an important factor affecting the initial construction scale. Generally, the first route will choose a traffic corridor with large passenger demand, which is mainly connected to the city center, important hub station or the connection between the main city and the new district. And timely adjustment of bus lines parallel to modern tram lines, residents living and working along the line and near the site need to choose new lines to meet travel needs. The induced passenger flow is the construction of a new type of transportation mode or improvement of service levels, improving the convenience of residents' travel, stimulating the travel needs of some residents, and causing changes in the travel behavior of the original travelers. These changes are directly reflected in the generation of new traffic demands, changes in travel time, increasing in travel times, and travel distance. For example, before the opening of the new line, due to the lack of convenient transportation, the surrounding residents would choose to shop in a more recent shopping area. When the line is opened, residents will travel more profitably and travel time will be shortened. Some residents will choose to shop in a large shopping area, which will increase the traffic of trams. The transferred passenger flow is due to the safety, convenience, improved service level, and travel time savings of the tram, which makes some passengers who choose other modes of transportation transfer to the tram. It does not change the total flow of passengers throughout the transportation system. Various factors affect the passenger flow of trams. The main influencing factors include the intensity of land development along the route [10,37], service level, fair, and transfer efficiency [9]. Land development along the line has a greater impact on passenger flow. The service level has the most obvious impact in the initial operational stage, which is an important guarantee for the trams to attract passengers. The passengers are sensitive to the level of fares, and therefore, a reasonable ticket price affects residents' choice of trams. Efficient transfer efficiency can quickly collect and distribute passengers, increasing the attractiveness of trams, and fully utilize the role of trams to ensure the efficiency of passengers.

System dynamics represents the structure of the system feedback through causal loop diagrams, including positive and negative feedback. Positive feedback loop causes enhanced changes, while negative feedback loop is to achieve a balance. The positive sign in the causal loop diagram indicates that the variable pointed to by the arrow will increase (or decrease) as the variable of the arrow source increases (or decreases). The negative sign indicates the opposite relationship between the variables. The feedback in the system reflects the causal relationship between variables. In the system dynamics model, causal loop diagrams are tools that reflect the relationship between variables in the systems. In the causal loop diagram, the causal relationship between variables is represented by a causal chain, and multiple causal chains close to form a causal loop diagram.

Based on the theory of system dynamics, according to the characteristics of passenger flow in the early stage of trams, after analyzing influencing factors, a causal relationship loop diagram between variables is drawn, as shown in Figure 3. Figure 3 demonstrates the causal relationship between passenger flow of trams and land development, ticket price, service level, and transfer efficiency. Four feedback loop diagrams are included: the causal relationship between passenger flow and land development (loop 1), passenger flow - fare causality (loop 2), passenger flow - service level causality (loop 3), and passenger flow - transfer efficiency causality (loop 4). The detailed information of the four feedback loop diagrams is given as follows.

Loop 1 shows the positive feedback relationship between passenger flow and land development intensity along the line. That is, land development intensity along the line $\rightarrow+$ coverage rate of the population $\rightarrow+$ population mobility $\rightarrow+$ trend growth passenger flow $\rightarrow+$ passenger flow. The nature of land use is diverse, especially for commercial land, office land, and residential land, and the population coverage of high-density land development is large and concentrated. When the accessibility of a station is high, the mobility of the population is 


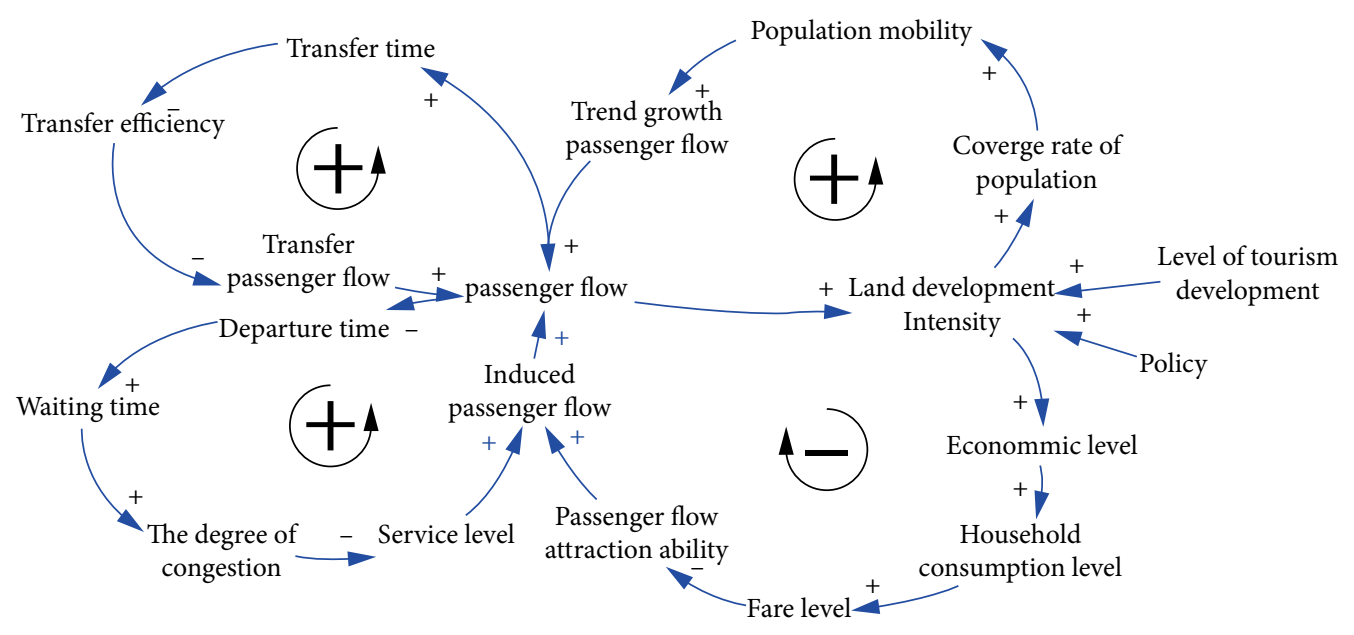

FIgURE 3: Passenger influencing variable causal loop diagram.

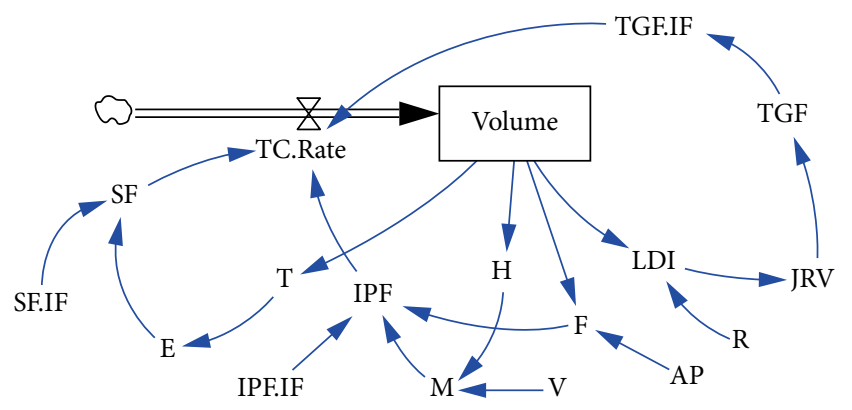

FIGURE 4: Modern tram passenger flow variable flow diagram.

increased, and the passenger flow is increasing. In addition, the strong support of government policies and the development of tourism along the line will also bring a trend of growth in passenger flow.

Loop 2 shows the negative feedback relationship between passenger flow and fare. That is, land development intensity along the line $\rightarrow+$ economic level $\rightarrow+$ household consumption level $\rightarrow+$ fare level $\rightarrow-$ passenger flow attraction ability $\rightarrow+$ the induced passenger flow $\rightarrow+$ passenger flow. Passenger flow is more sensitive to fare levels. If the fare is too high, the tram itself is not attractive, and it will inevitably lose a large number of passengers who transfer to other transportation modes. If the fare is too low, on the one hand, the rapid growth of passenger flow at the initial stage will increase the congestion of trams as well as platforms which will result in reduced service level; on the other hand, the operating company's financial situation will deteriorate and financial pressure will be brought to the government.

Loop 3 shows the positive feedback relationship between passenger flow and service level. That is, departure interval $\rightarrow+$ waiting time $\rightarrow+$ the degree of congestion $\rightarrow-$ service level $\rightarrow+$ the induced passenger flow $\rightarrow+$ passenger flow $(\rightarrow-$ departure interval). The impact of service level on passengers is mainly the departure interval. Longer departure intervals result in extended passenger waiting times. In the early operational stage, the stability of the system is weak, resulting in lower service levels and affecting attraction to passengers. The service level has a significant impact on passenger flow in the early operational stage.

Loop 4 shows the positive feedback relationship between passenger flow and transfer efficiency. That is, passenger flow $\rightarrow+$ transfer time (including on and off time, walking time, waiting time) $\rightarrow-$ transfer efficiency $\rightarrow-$ transfer passenger flow. Since the direct coverage region of a single-line is limited, transfer of other modes (including walking, bicycle, car, and bus) is very important to enlarge the influence area and give full play to the advantages of medium-sized passenger traffic. The effective attraction of the station to the longdistance passenger flow also depends on the transfer of these transportation modes.

3.3. Modeling. The causal loop diagram does not distinguish stocks and flow, i.e., only the cumulative and changing variables are in the system. The causal loop diagram is intuitive. But when the model needs to continue the quantitative model, the variables need to be distinguished and a stockflow graph is drawn based on the causal loop diagram so that the mathematical relationship between variables can be established. Based on the system dynamic, the Vensim is used. The passenger flow is used as a state variable, and the passenger flow changing rate is rate variable. Four factors (i.e., intensity, service level, fare, and connection transfer efficiency) are auxiliary variables. The framework of the model is depicted in Figure 4.

Note: Volume - passenger flow; TC. Rate - change rate of passenger flow; LDI - land development intensity along the line; R - plot ratio; JRV - posts within the affected area of the station/resident population /tourists; TGF - trend growth passenger flow; TGF. IF - influencing factors of trend growth passenger flow; F - fare; AP - alternative bus product price; IPF - induced passenger flow; IPF.IF - influencing factors of induced passenger flow; $\mathrm{H}$ - departure interval; $\mathrm{M}$ - service level; V - travel speed; $\mathrm{T}$ - transfer time of station; $\mathrm{E}$ - transfer efficiency of station; SF - transfer passenger flow; SF.IF influencing factors of transfer passenger flow.

For the influencing factors of the growth of modern tram passenger flow and the system dynamics equation, refer to the 
literature $[31,38]$. The main equations in Vensim include the equation of state, rate equation and auxiliary equation.

In the following equation, the state variable Volume is the variable that ultimately determines the behaviors of the system, which is directly changed by the rate:

$$
L: \text { Volume.k = INTEG(TC.rate), }
$$

where, INTEG represents the accumulated variable.

In the rate equation $R$, rate variable TC.Rate changes under the influence of trending passenger flow TGF, induced passenger flow $I P F$, and transfer passenger flow $S F$. The following equation is used to reflect the input of the state variables and output speed:

$R:$ TC. Rate $=\frac{[(T G F . j * T G F . I F+I P F . j * I P F . I F+S F . j * S F . I F)-T G F . k * T G F . I F+I P F . k * I P F . k * I P F . I F+S F . k * S F . I F]}{\text { Volume.k}}$.

The auxiliary equations constructed by auxiliary variables and constants reflect the process of information flow in the system and the behavioral changes of the simulation system under different conditions. The floor area ratio $R$ is the ratio of the total floor area to the total land area within a certain range (500 $\mathrm{m}$ is used in this study) [9]. Trend growth passenger flow is calculated by the number of jobs within the affected area of the station + permanent population + the number of visitors - the number of people working at home. Induced passenger flow refers to the flow of passengers attracted by service level and reasonable fare. Transfer of passenger flow refers to the flow of passengers transferred from other modes of transportation due to the increase in the efficiency of site transfer and transfer. The mathematical relationship between the variables in the model is given as follows:

$$
\left\{\begin{array}{l}
A_{1}: R=\frac{\left(\alpha F_{1}+\beta F_{2}+\mu F_{3}+\gamma F_{4}\right)}{S} \\
A_{2}: T G F=J R V-P \\
A_{3}: I P F=\lambda_{1} \text { Fare }+\lambda_{2} M=\lambda_{1} A P+\lambda_{2} M \\
A_{4}: M=\alpha_{1} V+\alpha_{2} H \\
A_{5}: T=t_{1}+\frac{H}{2}+\frac{L}{V} \alpha \\
A_{6}: E=\frac{S F}{T}
\end{array}\right.
$$

where, $L$ is the equation of state, $R$ represents the rate equation, $A$ denotes the auxiliary equation, $j$ and $k$ represents the current and previous moment, respectively; $F_{1}, F_{2}, F_{3}, F_{4}$ are the area of commercial, residential, industrial and scenic sites along the tram; $\alpha, \beta, \mu, \gamma$ are the weight of different types of buildings; $P$ denotes the number of people working at home along the line; $\lambda_{1}, \lambda_{2}$ is the weight of $\mathrm{A} * \mathrm{P}$ and $M$, respectively; $\alpha_{1}, \alpha_{2}$ are the impact factors; $t_{1}$ represents the time getting on/off trams; $K$ is the length of the transfer channel; $V_{\alpha}$ is the average speed of passengers.

\section{Data Description}

4.1. Investigation Scheme. This study mainly adopts RP and SP survey methods. The fact-based traffic RP survey mainly includes gender, age, transportation mode, income, travel purpose, travel time, and origination. The SP survey mainly includes changes in the willingness to travel after the operation of trams. The paper selects the Tram Line 1 in Huai'an City, Jiangsu Province in China as the case study. Unlike other cities, this tram line is the first line in China that runs through the core area of the city. The length of this line is $20.3 \mathrm{~km}$, connecting the core area of the city and the economic development zone. The total number of stations along this line is 23. From May to July 2016, investigators were organized to conduct questionnaire surveys in Huai'an. A total of 300 questionnaires were distributed. 300 questionnaires were retrieved, and the recovery rate is $100 \%$. There are 294 valid questionnaires, accounting for $98 \%$ of the questionnaires. At the same time, in order to ensure the validity of the survey, investigators conducted in-depth interviews with passengers and staff before the formal survey. The authors have revised the design of the questionnaire and the selection of variables. Key survey questions are listed in Table 1.

In order to obtain relevant passenger flow data, the manual survey is used. 10 people were assigned to perform the survey. When the vehicle passed a certain site, investigators recorded the site name, arrival time, and departure time of the trams. In order to make the data more consistent, the survey time was three working days from June 19 to 21,2016 , covering 189 shifts. No obvious weather changes and unexpected situations during the investigation.

Qualitative questionnaires are quantified, raw data were collected. The incomplete, inconsistent, and non-influential data were excluded, and validity data were analyzed to prepare for subsequent model simulation.

4.2. Parameters Determination. The driving interval $H$ of the initial operation of the line is $8 \mathrm{~min}$, and the running time of the line is about 1-hour. In May 2016, passenger flow volume was 360,000 passengers per month. The initial passenger volume Volume is $_{0} .3$ million passengers. Travel speed $V$ is $18-25 \mathrm{~km} / \mathrm{h}$, and passenger's average speed is 1.4 $\mathrm{m} / \mathrm{s}$. According to the questionnaire survey, the number of passengers belonging to the trend growth is 176-passengers, accounting for $60 \%$. The number of people involved in the induced passenger flow is 94-passengers, accounting for $32 \%$; the number of passengers belonging to the transfer is 24-passengers, accounting for $8 \%$. Thus, the value of TGF.IF, IPF.IF, and SF.IF is $0.6,0.32$, and 0.08 , respectively. There are signal priority facilities at each intersection, and the fare is 2 $\mathrm{RMB}$. Most of the sites are swiping or coin-operated on the train, and some sites are swiping or coin-operating during the peak hours. According to the collected data, $12 \%$ of the population choose to take a tram because of the alternative price of public transport along the route. $20 \%$ of the people who choose to take the tram are the service level, mainly the departure interval. As a result, the weight of $\lambda_{1}, \lambda_{2}$ is set to be 0.12 and 0.2 , respectively. According to the Huaian City Master Plan (2009-2030) and the Huaian Controlled Detailed Planning Management Measures, the $P$ value is determined to be $50,000 . F_{1}, F_{2}, F_{3}, F_{4}$ are $5.28,7.67,5.75$, and 1.3 , respectively. $\alpha, \beta, \mu, \gamma$ are $0.26,0.39,0.27$, and 0.08 , respectively. Since the 
TABLE 1: Key questions in the study of factors affecting passenger flow of trams.

(1) The purpose of your travel:

$\begin{array}{llll}\text { (A) Commute } & \text { (B) School } & \text { (C) Shopping } & \text { (D) Travel }\end{array}$

(E) Others

(2) The travel mode you usually use:

$\begin{array}{llll}\text { (A) Walking (B) Bicycle } & \text { (C) Car } & \text { (D) Bus }\end{array}$

$\begin{array}{lll}\text { (E) Tram (F) Taxi (G) Others } & \end{array}$

(3) The reasons you choose trams for travelling:

(A) Economical and practical (B) Rapid and convenient

(C) Punctual and reliable (D) Safety and comfortable

(E) Others

(4) The waiting time of trams:

$\begin{array}{llll}\text { (A) } \leq 5 \mathrm{~min} & \text { (B) } 5-10 \mathrm{~min} & \text { (C) } 10-15 \mathrm{~min} & \text { (D) }>15 \mathrm{~min}\end{array}$

(5) Acceptable fare of trams:

$\begin{array}{llll}\text { (A) } 1 \mathrm{RMB} & \text { (B) } 2 \mathrm{RMB} & \text { (C) } 4 \mathrm{RMB} & \text { (D) } 8 \mathrm{RMB}\end{array}$

(E) $10 \mathrm{RMB}$

(6) Transfer mode of trams:

$\begin{array}{llll}\text { (A) Bus (B) Bicycle sharing (C) Walking (D) Car } & \text { (D) }\end{array}$

(7) What do you think are the factors that affect the passenger flow of trams?

$\begin{array}{lll}\text { (A) Fare (B) Service level (C) Transfer efficiency } & \text { (C) Others }\end{array}$

(D) Others

initial operational period of rail transit is normally 5-7 years, the system simulation time is set from 2016 to 2022 .

\section{Simulation Analysis}

5.1. Model Validation. In order to ensure the validity and availability of the developed model as well as the causal relationship between various factors, model validation is performed (including sensitivity test and extreme condition test). The sensitivity test is described as follows. Sensitivity testing is to test whether the corresponding changes in other variables are reasonable when the value of variable changes, especially, for parameters with low estimation accuracy. Through sensitivity testing, the change of the simulation results of a model can be understood due to the change in the value of variables. Extreme condition testing is mainly used to examine whether the equations are stable and reliable. When the values of parameters are set to be the extreme value (i.e., maximum or minimum value), whether or not the model can reflect the changing law of the real system in any extreme case is explored.

5.1.1. Sensitivity Test. According to previous analysis, four variables (i.e., ticket price, land development intensity, service level, and transfer efficiency) contribute to passenger flow during the initial operational stage. Through sensitivity test, whether these variables have a significant impact on passenger flow can be observed. As shown in Table 2, all the four variables affect the passenger flow significantly. There is no abnormal reaction in the behavior of the model due to minor changes in the variable parameters. Among these variables, the sensitivity value of land development intensity is 2.1351 , which indicates it has the most significant impact on passenger flow. The sensitivity value of service level is greater than that of the ticket price. The possible reason is that residents have a lower sensitivity to the ticket price and higher sensitivity to time with the improvement in economic level.

5.1.2. Extreme Condition Test. According to survey results, the extreme values of variables or parameters in the developed model are set and tested. The range of plot ratio is between 0.1 and 10 . The maximum and minimum values of the departure interval are 1 and 45, respectively. The maximum and minimum values of ticket price are respectively set as 1 and 15 . The value of transfer efficiency is between 0.1 and 10. Testing results (see Figure 5) demonstrate that the passenger flow decreases dramatically when the plot ratio is set as 0.1 ; when plot ratio is 10 , the passenger flow increases rapidly. Similar results can be observed for the other three variables. For example, when the value of transfer efficiency is 0.1 , there is a significant growth in passenger flow as the year increases; however, when it is set to be 10 , due to the high requirements for transfer efficiency, passenger flow decreases. In short, all the testing results are in line with the actual situation, and therefore, the model is stable and reliable.

\subsection{Impact of Different Factors on Passenger Flow}

5.2.1. Impact of Land Development Intensity. Plot ratio is an important indicator for measuring the strength of land development. In Figure 6, the relationship between the plot ratio around the tram station and daily average passenger flow is presented. The simulation results demonstrate that the trend of plot ratio is generally consistent with the trend of daily average passenger flow at each station. Daily average passenger flow decreases as the plot ratio increases. In the cities in China where trams have been operated, most lines are operated in the new urban area. However, the tram passes through the downtown area in Huaian. Typically, a large amount of commercial and office land increases the mobility of the population forming the increased tread of passenger flow. For example, the Stadium Station-Shuidukou Square Station is located in the downtown area. There are many commercial lands, large shopping malls, and entertainment facilities which attract a large number of passengers. Government Affairs Center Station-Xiamen Road Station is a general office land where is suitable for large exhibitions. Thus, large passenger flow is easily formed as well. Ba Tingqiao Station-Hexia Station is the transition section. On the west side of the line is the Li Canal, which forms a sightseeing corridor, and the east side is the residential area, but it is in the construction process. Liziba Station-Nanmen Station is a suburb of Huaian dominated by industrial areas. It should be noted that the Golden Plaza is built around the Liziba Station, and commercial consumption facilities have some agglomeration effect on passenger flow. The Memorial Hall is surrounded by tourist attractions. Even though the plot ratio is low, the Memorial Hall, Ancient City Walls, and other attractions attract a large number of tourists. In addition, this line is currently used for medium and long distance passenger flow. As can be seen in Figure 6, the daily average passenger flow curve is concave. Passenger flow is basically concentrated at both ends of the line.

5.2.2. Impact of Service Level. The departure interval is an important indicator to measure the service level, and it is also 
TABLE 2: Sensitivity test table.

\begin{tabular}{lcccccc}
\hline Parameters & $-10 \%$ & $-5 \%$ & 0 & $5 \%$ & $10 \%$ & Sensitivity value \\
\hline Ticket price & -0.0716 & -0.0629 & 0 & 0.0374 & 0.0414 & 0.2370 \\
Land Development Intensity & -0.3219 & -0.1542 & 0 & 0.0655 & 0.1048 & 2.1351 \\
Service level & -0.1043 & -0.0915 & 0 & 0.0433 & 0.0687 & 0.4060 \\
Transfer efficiency & -0.0055 & -0.0037 & 0 & 0.0039 & 0.0051 & 0.0790 \\
\hline
\end{tabular}

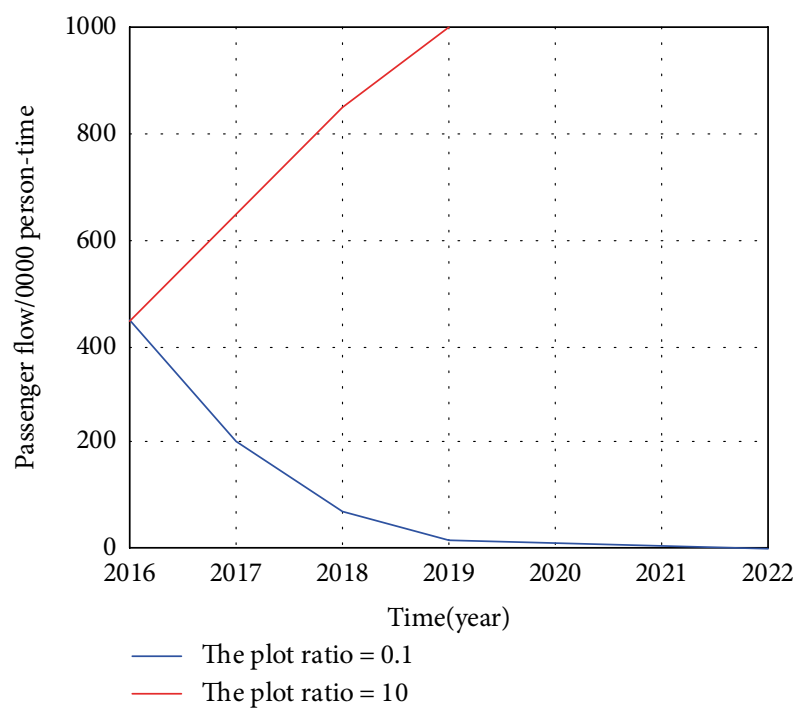

(a)

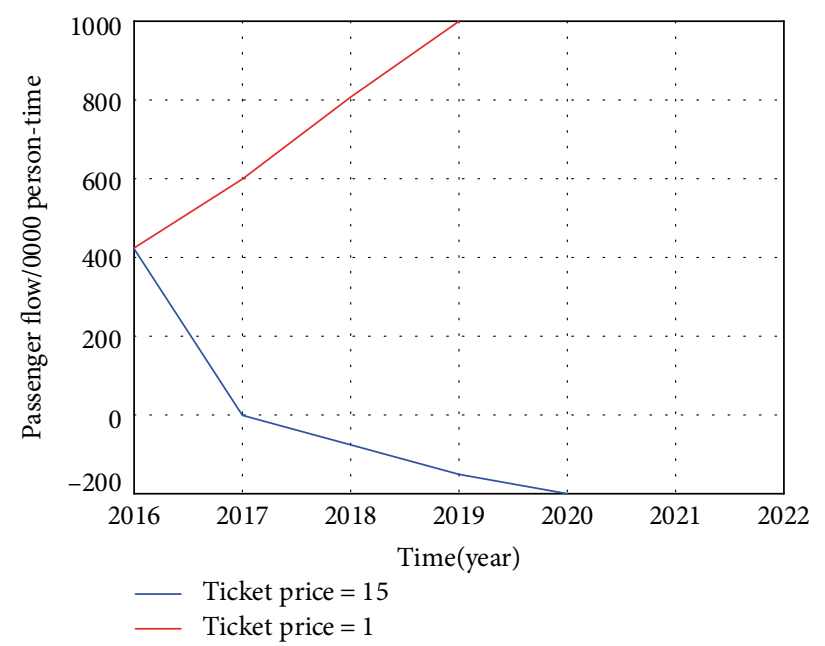

(c)

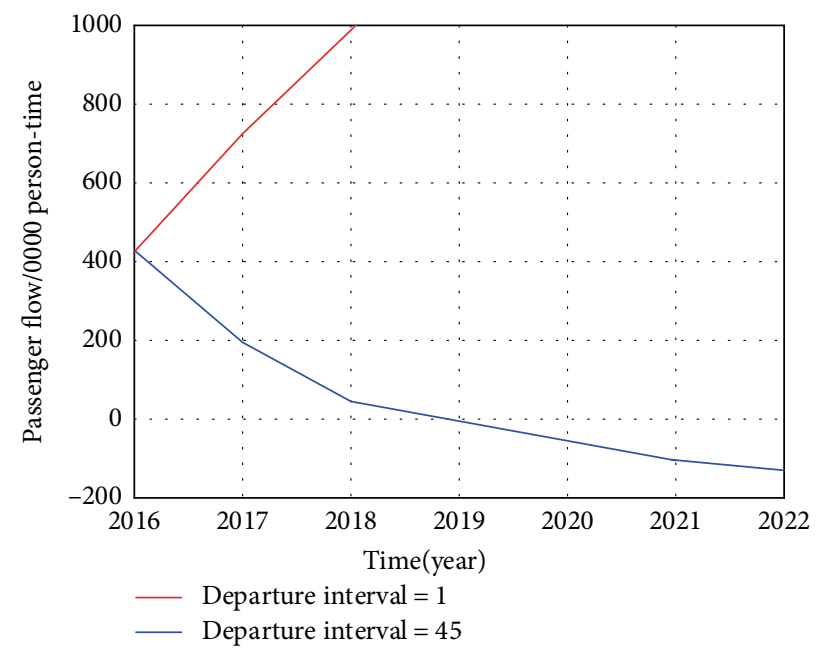

(b)

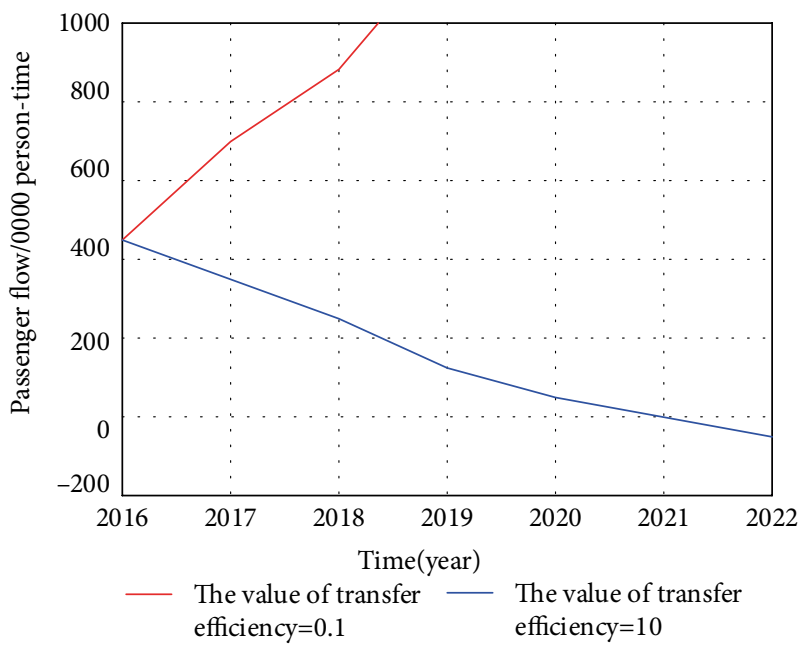

(d)

FIgURE 5: Extreme conditional tests for each variable. (a) Extreme condition test for plot ratio, (b) Extreme condition test for departure interval, (c) Extreme condition test for ticket price, and (d) Extreme condition test for transfer efficiency.

an indicator reflecting the attraction of passenger flow on the tram line. According to statistics, the initial departure interval of tram in Nanjing Hexi was $20-25$ min, and the current interval during peak hours is shortened to 12 minutes. The initial departure interval of Suzhou Tram was $15 \mathrm{~min}$, and the interval is reduced to $8 \mathrm{~min}$ during the peak periods. The interval of trams during the trial operation period in Huaian is $14-15 \mathrm{~min}$. The departure interval is currently adjusted to 7-8 min. The initial interval of trams in Songjiang, Shanghai was 10 minutes, and it is adjusted to $2-3$ min during the operational period. According to the survey, the departure interval is divided into 5 interval segments, i.e., $[0.5),[5,10)$, $[10,15),[15,20),[20,25]$. The average value of each interval is taken. When the departure interval is $2.5 \mathrm{~min}, 7.5 \mathrm{~min}, 12.5$ min, $17.5 \mathrm{~min}, 22.5 \mathrm{~min}$, the impact of different departure interval on passenger flow is presented in Figure 7 . When the departure interval is $2.5 \mathrm{~min}$ (i.e., [0.5)), passenger flow grows rapidly. However, as the year increases, its growth rate declines, due to the reason that the excessive growth of passenger flow will bring a series of problems. If the departure interval is 7.5 




FIGURE 6: The impact of service level on passenger flow.

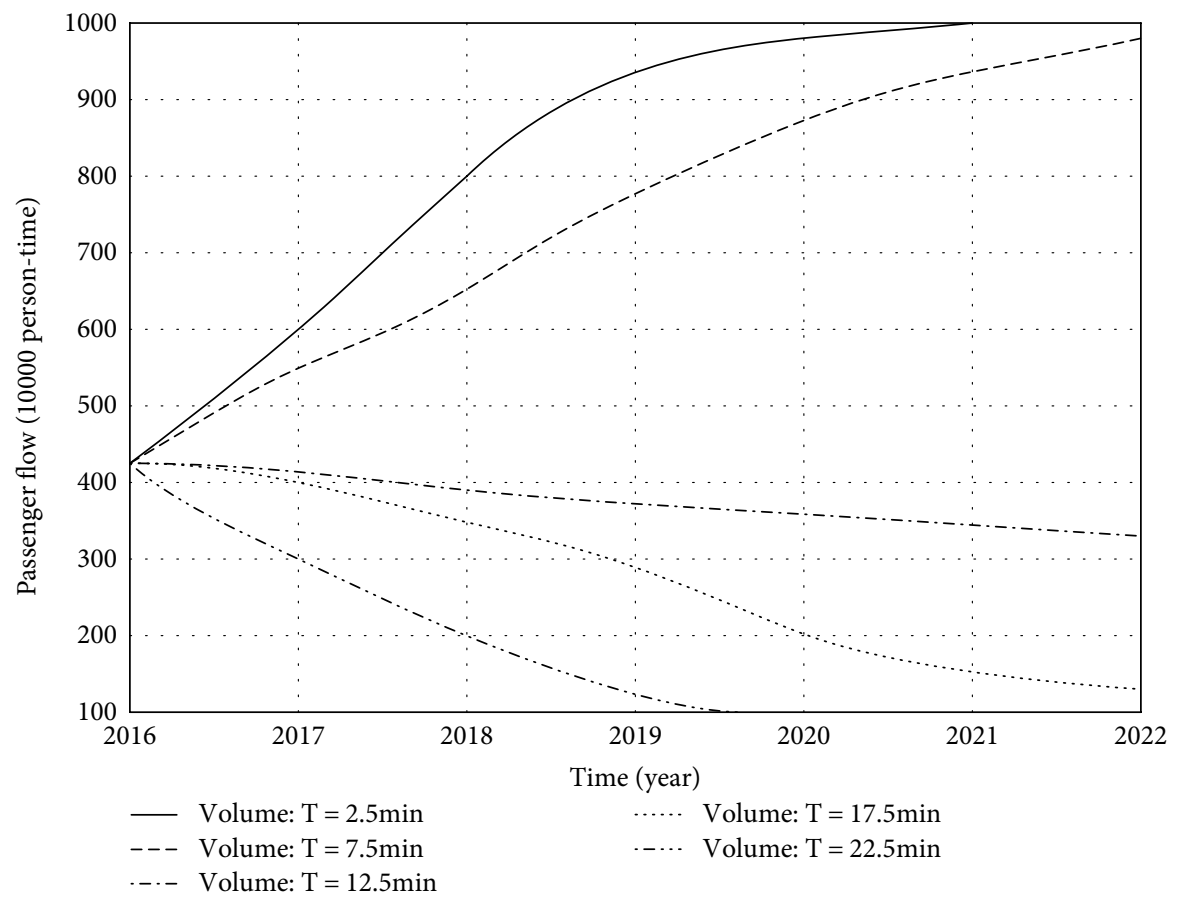

FIGURE 7: The impact of service level on passenger flow.

min (i.e., $[5,10)$ ), passenger flow grows steadily. However, due to the increase in passengers' travel time requirements, it leads to slow growth. When the departure interval is $12.5 \mathrm{~min}$ which is greater than $10 \mathrm{~min}$, the tram is almost unattractive to passengers. It can even lead to the loss of passengers and result in negative growth. One can clearly see from Figure 7 that $10 \mathrm{~min}$ is the maximum limit of passengers' psychological tolerance. Service level has the most obvious impact on the initial operation period. The function of the tram system is still unstable at the beginning of the operation. Therefore, it has a 


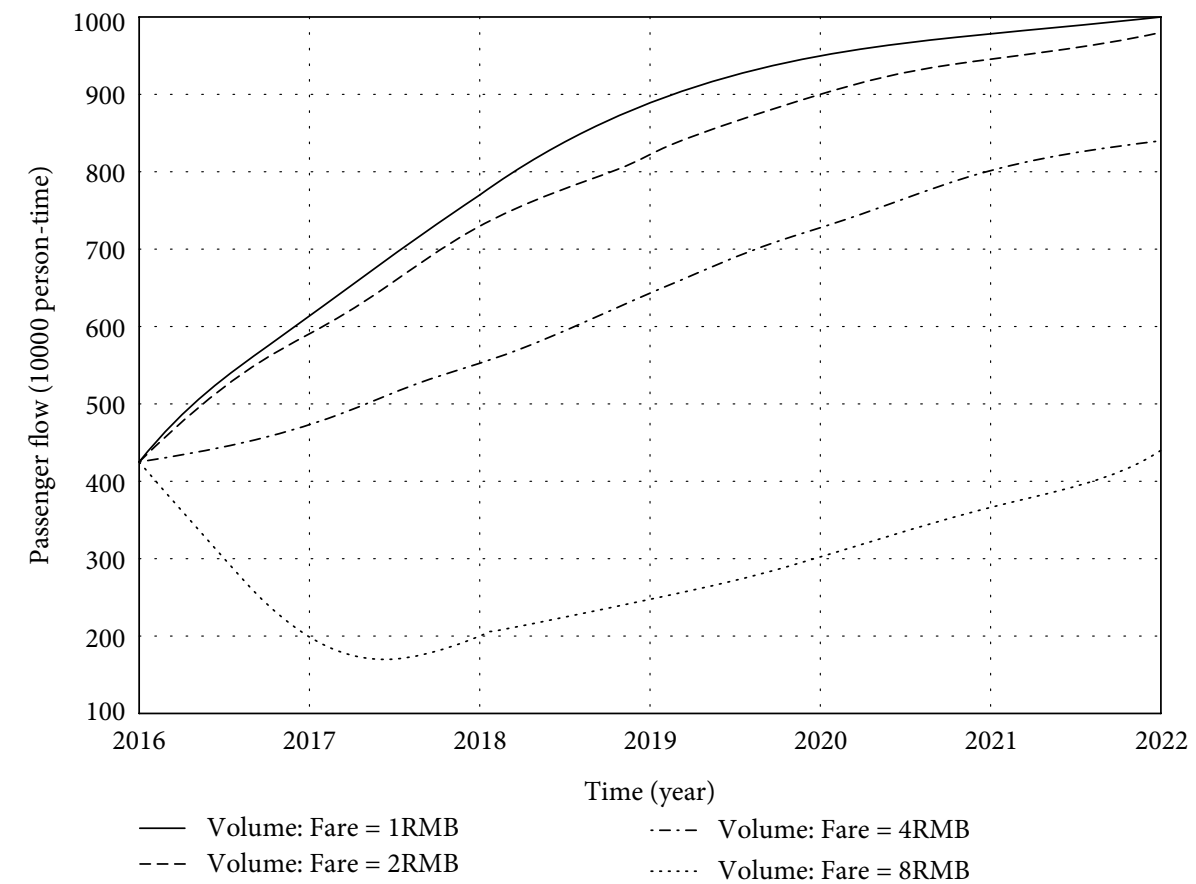

Figure 8: The impact of service level on passenger flow.

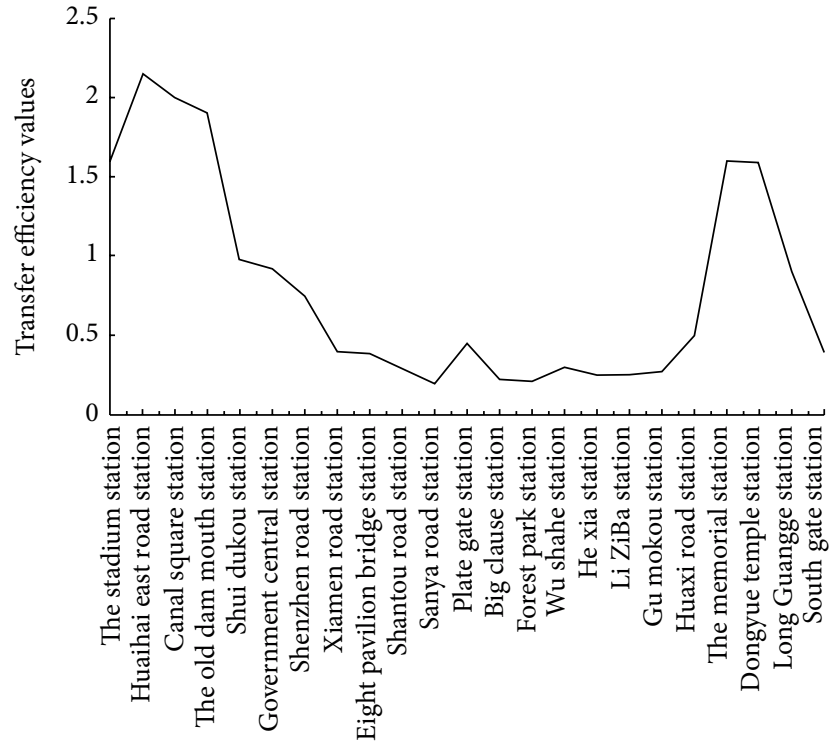

FIGURE 9: Transfer efficiency values for each station.

negative impact on operations for a long period of time, which makes the initial service level lower and affects passenger flow.

5.2.3. Impact of Fare. Figure 8 presents the impact of fare on passenger flow. When the fares are 1, 2, 4, and $8 \mathrm{RMB}$, respectively, the passenger flow curves from 2016 to 2022 are depicted. As shown in Figure 8, the fare of $2 \mathrm{RMB}$ is the psychologically reasonable fare of passengers. When the fare is lower than the psychological price, the passenger flow grows rapidly. Due to the limited operation resources (including the limited car loading and the total capacity), passenger flow grows slowly. When the fare is higher than the psychological price, the passenger flow slows down. When the fare is too high (i.e., $\geq 8 \mathrm{RMB}$ ), the passenger flow shows a negative growth trend. Generally speaking, the fare is a key factor in attracting passengers based on the simulation results, particularly during the initial operational period. Too high fare makes the trams less attractive, which is not conducive to increasing passenger flow. The role of trams in increasing volume cannot be played, which will lead to the waste of newstyle traffic system resources. If the fare is too low, on the one hand, the rapid growth of passenger flow at the initial stage will increase the congestion of trams as well as platforms which will result in reduced service level; on the other hand, the operating company's financial situation will deteriorate and financial pressure will be brought to the government.

5.2.4. Impact of Transfer Efficiency. After field investigation, the number of connection points, connection distance, transfer time, average waiting time, and channel distance of buses, cars, and bicycles at various stations along the route are analyzed. The transfer efficiency of each station is estimated, as shown in Figure 9. The estimated values are divided into three intervals, i.e., $[0,0.8),[0.8,1.6)$, and $[1.6$, 2.4]. The average value of each interval is used to simulate the impact of transfer efficiency at the station on passenger flow, and the simulation results are presented in Figure 10. When the average transfer efficiency of a station is 2.0 (i.e., the efficiency value is $[1.6,2.4])$, such transfer efficiency effectively promotes a large increase in passenger flow from 2016 to 2020, and the passenger flow grows slowly after 2020 . When the average transfer efficiency is 0.4 (i.e., the efficiency value is $[0,0.8)$, the low transfer efficiency impedes passenger flow growth and leads to the loss of passengers. The initial 


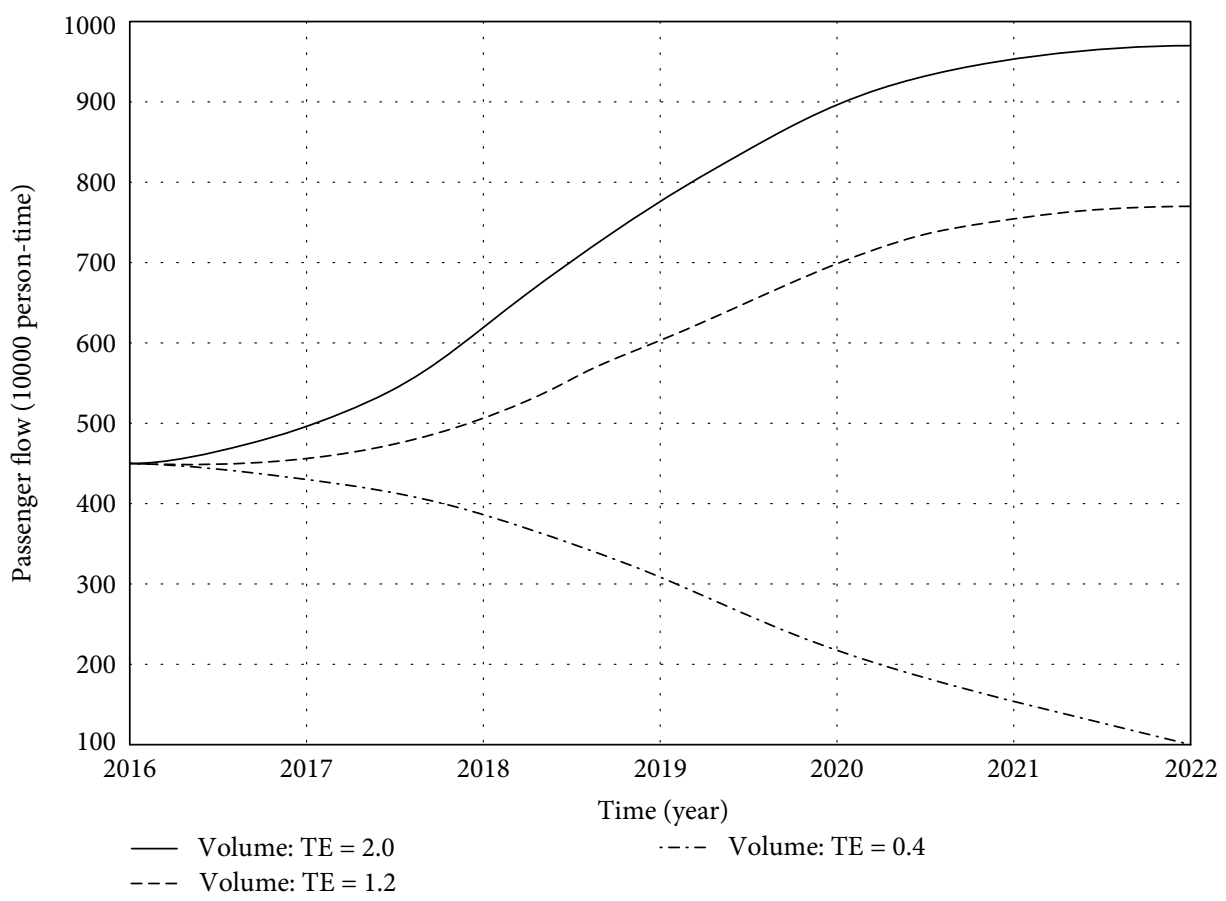

Figure 10: Influence of transfer efficiency on passenger flow.

operation of trams is usually single-line operation, and its impact is very limited, generally covering both sides of the line. Thus, it needs different transportation modes (including walking, bicycle, car, and bus) for transfer to increase the accessibility of trams.

\section{Discussion and Conclusion}

This paper studies the factors that contribute to the growth of passenger flow during the initial operational period of trams. Relevant policy recommendations for the promotion of passenger flows are designed, which are summarized as follows.

The intensity of land development affects the maximum population size within a certain area. The higher the intensity, the higher the passenger flow. The intensity of land development along the line should be positioned according to the type of function of the station. The high plot ratio is used for the development of land around urban stations (such as the Gymnasium Station-Shuidukou Square Station). Land development is dominated by commerce, including large shopping malls, pedestrian streets, business offices, leisure, and entertainment industries. The peripheral stations are suitable for medium and high-intensity development. For example, the east side of the Ba Tingqiao Station-Hexia Station of the line is a residential area. The construction speed should be accelerated so that the occupancy rate of the residential area should be improved, and the living facilities should be improved to increase passenger flow. In addition, the land plot ratio around the landscape station is low, but the connection facilities of the station and the scenic spot and the supporting commercial facilities should be improved to attract passenger traffic.
The departure interval has the most obvious impact on the passenger flow during the early operational stage. The departure interval of 10-min is the maximum limit of passengers' psychological tolerance based on the simulation results. Fluctuating departure intervals lead to low service levels. The intelligent electronic display screens should be installed at each station to play the interval between the trains and the arrivals information in real-time.

The strategies that can be used to enhance the attraction of trams to passengers should be developed during the initial operational stage. For example, a competitive fare policy should be developed considering passengers' ability to pay. According to this study, the fare of 2 RMB is the psychologically reasonable fare of the passenger. Such price of ticket is good for attracting more passengers and inducing passenger flow on weekdays.

When constructing trams, the connection and transfer facilities of various modes (e.g., electric vehicles, bicycles and private cars, taxis and regular bus stops) should be built simultaneously to satisfy the requirements for parking. In addition, according to the direction of the tram, some parallel bus lines should be canceled or shortened. Moreover, the bus lines that are radiated to the main stations of the trams in all directions should be added so that a supply and demand relationship can be formed.

The paper takes the modern tram line in Huaian, Jiangsu Province, China as the case study. The system dynamics method is employed to explore the influencing factors of passenger flow. The results demonstrate that four variables have a significant impact on passenger flow, including land development intensity, fares, service levels, and transfer facilities along the line. The intensity of land development has the most significant impact on passenger flow. Passenger flow increases with the increase of plot ratio. Commercial consumption 
facilities have a greater effect on passenger flow, and the scenic spots along the line attract passengers. Due to the improvement of residents' economic level, passengers' sensitivity to fares is reduced, and time requirements are high. The impact of service level on passenger flow is higher than that of fares on passenger flow. The fare of $2 \mathrm{RMB}$ is the psychologically reasonable price of the passengers. When the fare is too high, negative growth in passenger flow is observed. High transfer efficiency will lead to a large increase in passenger flow, but too low transfer efficiency will hinder the growth of passenger flow, resulting in loss of passengers. The data samples collected in the paper have limitations. It is necessary to collect multiple city data and improve data samples.

\section{Data Availability}

The Survey.xls data used to support the findings of this study have been deposited in the repository of https://pan.baidu. com/s/1Z-2yTD1Tfg9V18HCXGGt5w for public access.

\section{Conflicts of Interest}

The authors declare that there are no conflicts of interest regarding the publication of this paper.

\section{Acknowledgments}

This research is supported by the Projects of the National Natural Science Foundation of China (51208256) and the National Key R\&D Program of China (2017YFB1201004). We would like to thank the editor and anonymous reviewers for providing insightful comments to improve our paper.

\section{References}

[1] H. Hondius, "The development of low-floor trams," Journal of Advanced Transportation, vol. 20, no. 1, pp. 79-102, 1993.

[2] B. Gunnarsson and A. Löfgren, "Light rail: experiences from Germany, France and Switzerland Ph.D. Thesis," Lulea University, Sweden, 2001.

[3] RUDOLPHIM, "A study of the possibility and potential effects of a tramway tunnel construction in Gothenburg City Ph.D. Thesis," Chalmers University of Technology, Sweden, 2012.

[4] Y. K. Li and Y. Y. Miao, "Enlightenment from the foreign modern tram construction and development," Urban Mass Transit, vol. 6, pp. 29-32, 2013, (in Chinese).

[5] J. G. Shi, Y. S. Sun, P. Schonfeld, and J. Qi, "Joint optimization of tram timetables and signal timing adjustments at intersections," Transportation Research Part C: Emerging Technologies, vol. 83, pp. 104-119, 2017.

[6] Centennial building network, "List of national urban tramway operation and construction line [OL]," http://www.wtoutiao. com/p/F16s1D.html.

[7] Mayor of London, “Tramlink User Guide," Mayor of London, London Tramlink, 2012.

[8] Y. S. Sun, J. G. Shi, and P. M. Schonfeld, "Identifying passenger flow characteristics and evaluating travel time reliability by visualizing AFC data: a case study of Shanghai Metro," Public Transport, vol. 8, no. 3, pp. 341-363, 2016.

[9] H. X. Pan, J. Li, Q. Shen, and C. Shi, "What determines rail transit passenger volume? Implications for transit oriented development planning," Transportation Research Part D: Transport and Environment, vol. 57, pp. 52-63, 2017.

[10] Y. Yuan, M. Yang, J. Wu, S. Rasouli, and D. Lei, "Assessing bus transit service from the perspective of elderly passengers in Harbin, China," International Journal of Sustainable Transportation, vol. 13, no. 10, pp. 176-776, 2019.

[11] X. Fu and W. H. Lam, "Modeling joint activity-travel pattern scheduling problem in multi-modal transit networks," Transportation, vol. 45, no. 1, pp. 23-49, 2018.

[12] Y. Ji, Y. Fan, A. Ermagun, X. Cao, W. Wang, and K. Das, "Public bicycle as a feeder mode to rail transit in China: The role of gender, age, income, trip purpose, and bicycle theft experience," International Journal of Sustainable Transportation, vol. 11, no. 4, pp. 308-317, 2017.

[13] C. Wang, Z. Ye, E. Chen, M. Xu, and W. Wang, "Diffusion approximation for exploring the correlation between failure rate and bus-stop operation," Transportmetrica A: Transport Science, vol. 15, no. 2, pp. 1306-1320, 2019.

[14] R. M. González, M. B. Eduardo, J. D. Juan, and E. Arnaldo, "Explanatory factors of distorted perceptions of travel time in tram," Transportation Research Part F: Traffic Psychology and Behaviour, vol. 30, pp. 107-114, 2015.

[15] L. Zhang, J. Lu, X. Yue, J. Zhou, Y. Li, and Q. Wan, "An auxiliary optimization method for complex public transit route network based on link prediction," Modern Physics Letters B, vol. 32, no. 5, Article ID 1850066, 2018.

[16] Y. Pan, S. Chen, F. Qiao, S. V. Ukkusuri, and K. Tang, "Estimation of real-driving emissions for buses fueled with liquefied natural gas based on gradient boosted regression trees," Science of the Total Environment, vol. 660, pp. 741-750, 2019.

[17] H. B. Zi, X. C. Guo, and J. Yang, "Research on application mode and regional applicability of modern trolley," Urban Mass Transit, vol. 18, no. 2, pp. 46-49, 2009, (in Chinese).

[18] N. A. Termida, Y. O. Susilo, and J. P. Franklin, "Observing dynamic behavioural responses due to the extension of a tram line by using panel survey," Transportation Research Part A: Policy and Practice, vol. 86, pp. 78-95, 2016.

[19] R. M. González, Á. S. Marrero, and E. Cherchi, “Testing for inertia effect when a new tram is implemented," Transportation Research Part A: Policy and Practice, vol. 98, pp. 150-159, 2017.

[20] J. Gadziński and A. Radzimski, “The first rapid tram line in Poland: How has it affected travel behaviours, housing choices and satisfaction, and apartment prices," Journal of Transport Geography, vol. 54, pp. 451-463, 2016.

[21] K. Chatterjee and K. R. Ma, "Modeling the timing of user responses to a new urban public transport service: application of duration modeling," Transportation Research Record: Journal of the Transportation Research Board, vol. 43, no. 1, pp. 64-72, 2007.

[22] D. Walton and S. Sunseri, "Factors influencing the decision to drive or walk short distances to public transport facilities," International Journal of Sustainable Transportation, vol. 4, no. 4, pp. 212-226, 2010.

[23] N. Paulley, R. Balcombe, R. Mackett et al., "The demand for public transport: the effects of fares, quality of service, income and car ownership," Transport Policy, vol. 13, no. 4, pp. 295-306, 2006.

[24] D. Huang, Z. Liu, P. Liu, and J. Chen, "Optimal transit fare and service frequency of a nonlinear origin-destination based 
fare structure," Transportation Research Part E: Logistics and Transportation Review, vol. 96, pp. 1-19, 2016.

[25] S. P. Shepherd, "A review of system dynamics models applied in transportation," Transportmetrica B: Transport Dynamic, vol. 2, no. 2, pp. 83-105, 2014.

[26] M. Besiou, O. Stapleton, and L. N. V. Wassenhove, "System dynamics for humanitarian operations," Journal of Humanitarian Logistics and Supply Chain Management, vol. 1, no. 1, pp. 78103, 2011.

[27] S. Liu, K. P. Triantis, and S. Sarangi, "A framework for evaluating the dynamic impacts of a congestion pricing policy for a transportation socioeconomic system," Transportation Research Part A : Policy and Practice, vol. 44, no. 8, pp. 596-608, 2010.

[28] H. X. Yang, J. D. Li, H. Zhang, and S. Q. Liu, "Research on urban traffic congestion control based on system dynamics," System Engineering Theory and Practice, vol. 34, no. 8, pp. 2135-2143, 2014.

[29] J. Li, W. W. Zhang, X. Y. Bai, S. M. Li, and K. J. He, "Research on influencing factors of emergency material transfer speed based on system dynamics," System Engineering Theory and Practice, vol. 35, no. 3, pp. 661-670, 2014.

[30] J. B. Zhao, W. Deng, Y. Song, and Y. R. Zhu, "What influences Metro station ridership in China? Insights from Nanjing," Cities, vol. 35, pp. 114-124, 2013.

[31] X. Y. Miao, H. Hu, and L. J. Gao, "Study on the growth of passenger flow in urban rail transit based on system dynamics," Railway Transport and Economy, vol. 34, no. 2, pp. 40-45, 2012, (in Chinese).

[32] P. Guo and R. H. Xu, "Study on the growth of passenger flow in urban rail transit based on system dynamics," Urban Rapid Rail Transit, vol. 19, no. 6, pp. 38-41, 2006, (in Chinese).

[33] J. He, C. Zhang, and W. Hang, "Complex transportation problems policy decision-making modeling based on the system dynamics method," China Communication Publishing, Beijing, 2016, (in Chinese).

[34] J. Zhang, "Study of the initial passenger flow evolution and its influential factors in urban rail transit -take xi'an as an example Ph.D Thesis," Chang'an University, Xian, China, 2017, (in Chinese).

[35] M. R. Liu, "Study on the impact of residents trip mode split when opening a new urban rail transit line Ph.D. Thesis," Beijing Jiaotong University, Beijing, China, 2015, (in Chinese).

[36] Y. P. Wang, "Study on urban rail transit passenger forecast and analysis Ph.D. Thesis," Chang'an University, Xian, China, 2011, (in Chinese).

[37] B. P. Y. Loo, C. Chen, and E. T. H. Chan, "Rail-based transitoriented development: lessons from New York City and Hong Kong," Landscape and Urban Planning, vol. 97, no. 3, pp. 202-212, 2010.

[38] Q. P. Wang, "System Dynamics," Shanghai University of Finance and Economics Press, SHANG Hai, 2009, (in Chinese). 


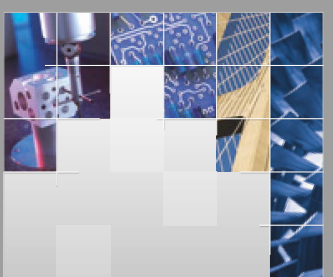

\section{Enfincering}
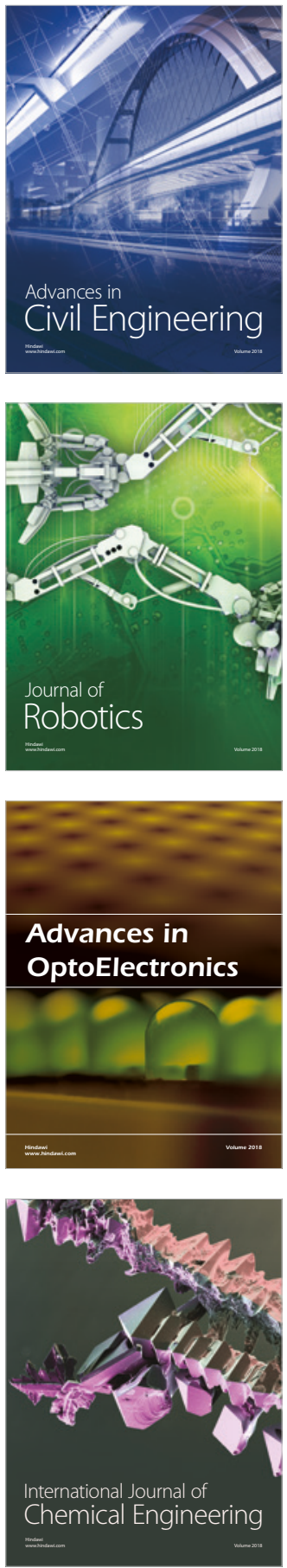



\section{Rotating \\ Machinery}

The Scientific World Journal

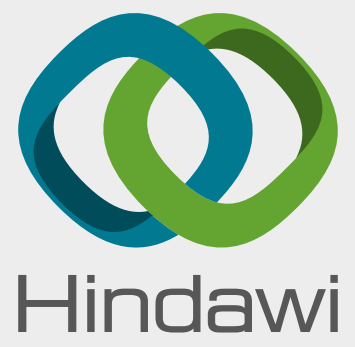

Submit your manuscripts at

www.hindawi.com
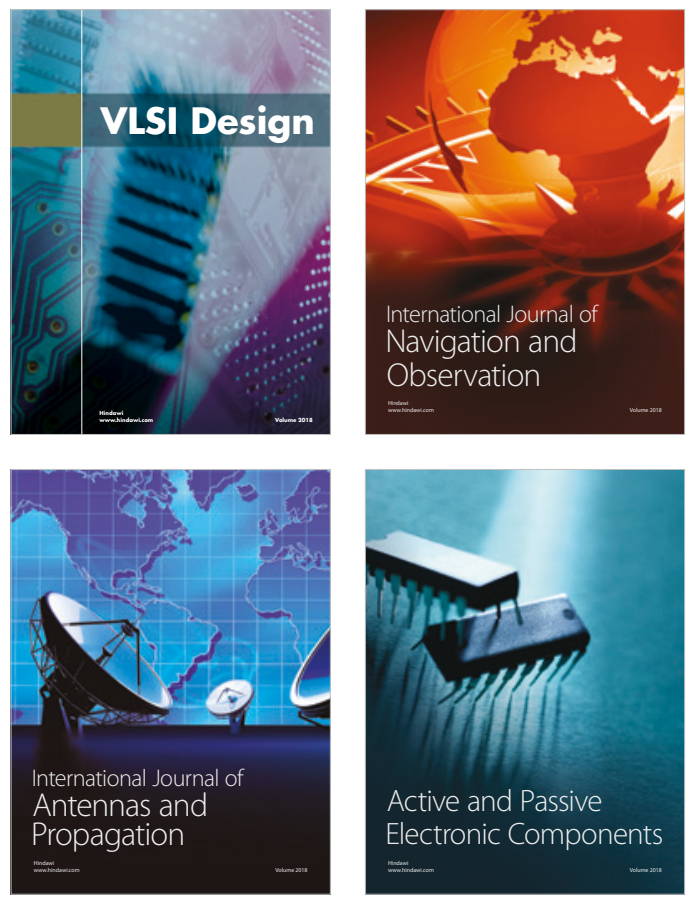
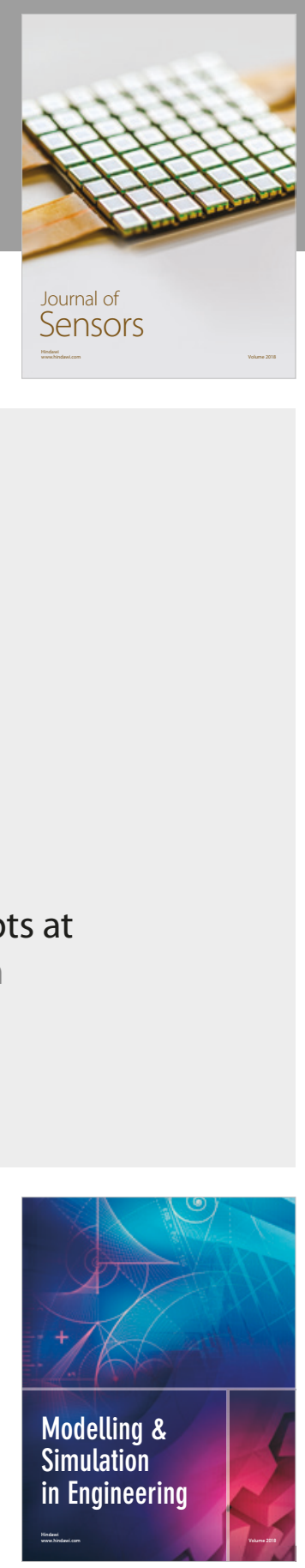

\section{Advances \\ Multimedia}
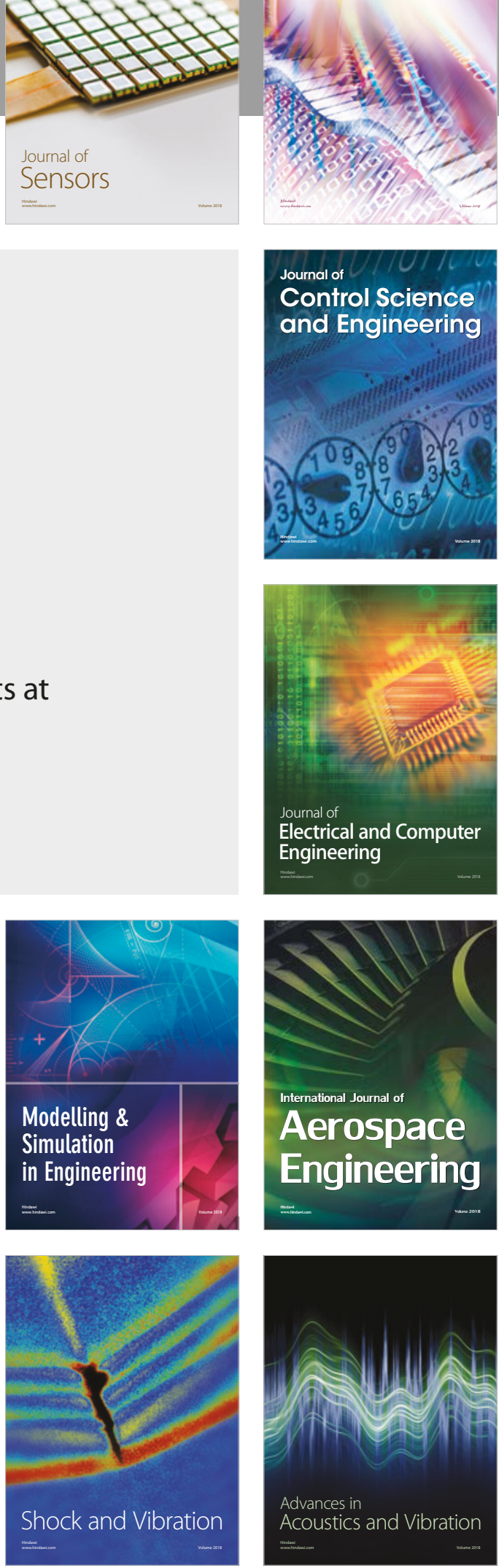